\title{
Using Advanced Simulation Techniques to Improve Industrial Controller's Dependability
}

\author{
Eurico Seabra, José Machado
}

University of Minho, School of Engineering, CT2M, Mechanical Engineering Department

\{eseabra,jmachado\}@dem.uminho.pt

\begin{abstract}
Modelica Modeling language is powerful and suitable for modeling mechatronic systems, being possible to interact different technological aspects and deal, simultaneously with different technologies (mechanical, electrical, pneumatic, hydraulic,...). In this paper it is discussed, in a case study, the possibility of using this language for modeling an automation system (controller and plant) in closed loop behavior and also in defining some parameters of the automation system in order to optimize some behavior aspects of the system as, for instance, the time cycle of the automation system. Some aspects relied with controllers dependability are also discussed and it is showed how Modelica modeling language can help controllers' designers improving controllers dependability, when are used Simulation Techniques.
\end{abstract}

\section{INTRODUCTION}

There is a rapidly increasing use of computer simulations in industry to optimize products, to reduce product development costs and time by design optimization, and to train operators. Whereas in the past it was considered sufficient to simulate subsystems separately, the current trend is to simulate increasingly complex physical systems composed of subsystems from multiple domains.

In such a complex industrial process, simulation tools are extremely useful since they can contribute to higher product quality and production efficiency in several ways. For example, modifications in a plant could be tested (both statistically and dynamically) in advance in a simulator saving much of the trial and error procedure that is used nowadays; the optimization of plant behavior parameters can be performed too. Besides, a dynamic simulator of the plant and of its control would allow for a thorough study of different control strategies, and would be an efficient way to tune controllers for new equipments. Finally, a simulation tool can also be a way of training not only the operators but also the production engineers and technicians. Some tools have been developed in order to simulate the behavior of automation systems (figure 1).

Graphical block diagram modeling is widely used in control engineering [1]. Some examples of languages and environments supporting this paradigm are Matlab/Simulink [2], MATRIXX/SystemBuild [3], HYBRSIM [4] and ACSL Graphics Modeller [5]. Block diagram modeling paradigm might be considered as a heritage of analog simulation [6].

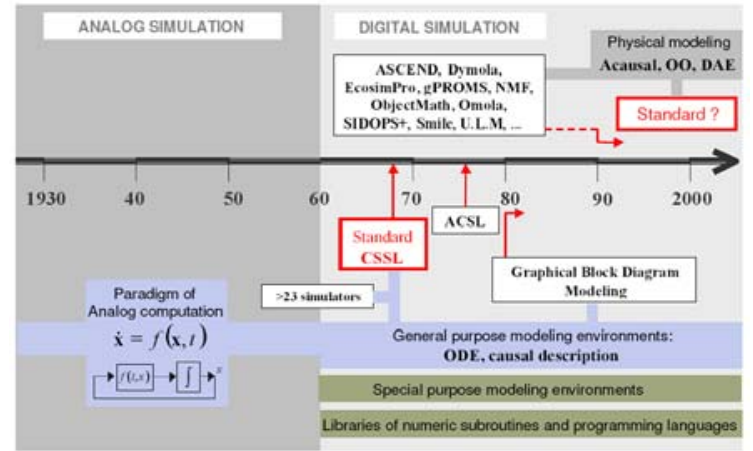

Fig. 1. Evolution of modeling and simulation tools.

In this paper it is presented a study and shown how Modelica modeling language can be used to optimize plant behavior parameters in order to guarantee the good and desired behavior for the system, in the shorter time cycle, combined with other aspects like energy consumption, for example.

To achieve the proposal goal, the section 2 is devoted to the presentation of Modelica modeling language and the Dymola Simulation environment; section 3 presents the case study that is the base for our study; section 4 discusses the mathematical modeling of the plant. Further, section 5 presents the Modelica model of the system (controller model coupled with plant model); section 6 discusses the obtained results concerning the defined plant behavior parameters to study and, finally, section 7 , presents some conclusions and future works, in this field.

\section{MODELICA AND DYMOLA}

In the few years of research in modeling and simulation, the concept of object-oriented modeling has achieved a big relevance. Several works have demonstrated how objected oriented concepts can be successfully employed to support hierarchical structuring, reuse and evolution of large and complex models independent from the application domain and specialized graphical formalism.

To handle complex models, the reuse of standard model components is a key issue. But in order to exchange models between different packages an unified language is needed. Modelica is an object-oriented, general-purpose modeling language that is under development in an international effort to introduce an expressive standardized modeling language, see [7] [8]. Modelica supports object-oriented modeling using inheritance concepts taken from computer languages such as Simula and $\mathrm{C}++$. It also supports non-causal modeling, meaning that model's terminals do not necessarily have to be 
assigned an input or output role. In fact, in the last few years it has been proved in several cases that non-causal simulation techniques perform much better than the ordinary objectoriented tools.

Modelica is a powerful programming language where equations are used for modeling of the physical phenomena. No particular variable needs to be solved for manually because the software Dymola [9] has enough information to decide that automatically. This is an important property of Dymola to enable handling of large models having more than hundred thousand equations. Modelica supports several formalisms: ordinary differential equations (ODE), differential-algebraic equations (DAE), bond graphs, finite state automata, Petri nets, etc.

\section{CASE STUDY DESCRIPTION}

The case study that is proposed as base for this work is inspired on the benchmark system proposed by [10].

Figure 2 illustrates an example of an evaporator system, which consists of two tanks, where an aqueous solution suffers transformations. In the first tank that solution should acquire a certain concentration through the heating of the solution using an electrical resistance $(\mathrm{H} 1)$ which provokes the steam formation.

Associated to the tank1 (figure 2) exist a condenser (C) responsible for the condensation of the steam that however it was formed. The cooling, in that condenser, it is done through the circulation of a cooling liquid (whose flow is measured by sensor FIS) that passes through the cooling circuit (if open the valve V13).

Associate to the tank1 there are a group of sensors: level sensors (maximum (LIS1) and minimum (LII1)), temperature sensor (acceptable maximum (TIS1)); sensor of conductivity (QIS) that is to indicate the desired concentration; they also exist several actuators: filling valve of the tank1 (V12), drain valve (V16) and emptying valve (V15), that it is also the filling valve of the tank2.

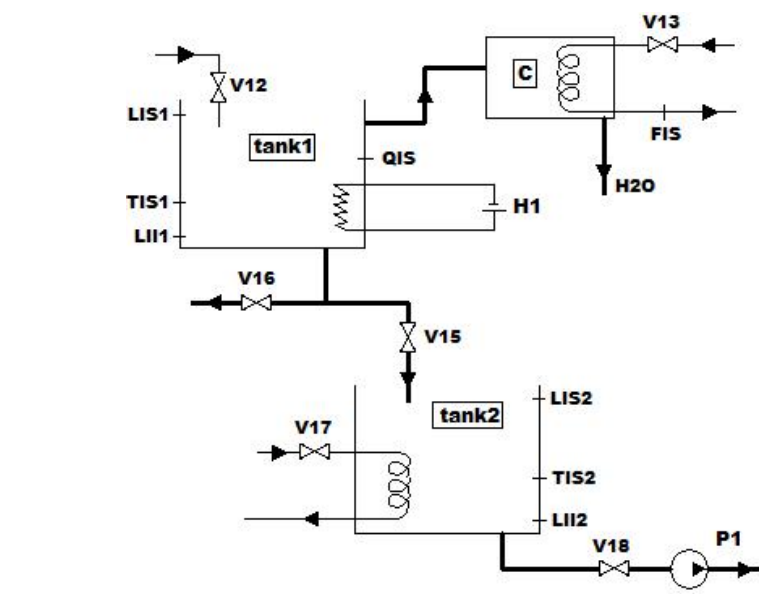

Fig. 2. Scheme of the entire evaporator system.

In the normal operation mode, the system works as follows.
The tank1 should be previously filled to its superior level with an aqueous solution by opening valve V12. When the tank1 is full, the heating system is switch on and also, in simultaneous, the cooling system of the condenser by opening valve V13. When it is formed steam, this condenses in the condenser $\mathrm{C}$. When the concentration desired in the tank 1 is reached, there are switch off the heating system and the cooling system of the condenser. Continuously the solution flows from tank1 into tank2, and it must be guaranteed that the tank 2 is empty. The transfer of the solution to the tank 2 is for a powder-processing operation that is not, here, described. For that powder-processing operation, there is necessary to heat the solution to avoid possible crystallization, and for that there are two approaches: it can heat until the temperature sensor of the tank2 indicates that the desired temperature was reached; or it can heat up for a certain time. Finally, the tank2 is emptied by the pump P1, if the valve V18 be opened.

On the other hand, in the possible failure operation mode, the system works as follows.

A possible failure scenario of the system happens when the cooling fluid flow in the condenser be to low (detected by sensor FIS). This implicates the increase of pressure and temperature in condenser $\mathrm{C}$ and tank1, if the heating system keep switch on (solution steam). It is necessary to guarantee that the pressure in the condenser $\mathrm{C}$ doesn't exceed a maximum value to avoid its explosion. For that, it should be guaranteed that the heating in the tank1 is switch off before the open of the safety valve (V16).

For this situation of failure operation, it should switch off the resistance $\mathrm{H} 1$ the more quickly possible, but tends in account that the solution doesn't crystallize, then that we are before a critical time. To switch off the resistance $\mathrm{H} 1$ they are considered two possibilities: through a time after sensor FIS to have detected reduced flow; or through the sensor of temperature TIS1 (due to the pressure and temperature are parameters that are directly related).

There are evidences that should be guaranteed, as for instance that the tanks should never overflow. After the failure situation occurs, all of the valves should be immediately closed.

\section{A. Controller Specification}

In order to guarantee the desired behaviour, the controller specification was developed according to IEC 60848 SFC specification.

The input and output variables of the controller which controls the process in closed-loop are presented and described in table 1.

The SFC specification of the controller behaviour (normal and failure modes) is presented in figures 3 and 4 .

TABLE I

INPUT/OUTPUT VARIABLES OF THE CONTROLLER

\begin{tabular}{|l|l|}
\hline \multicolumn{1}{|c|}{ Inputs } & \multicolumn{1}{|c|}{ Outputs } \\
\hline LIS1 - Superior level of the tank1 & $\begin{array}{l}\text { V12 - Solution entrance of the } \\
\text { tank1 }\end{array}$ \\
\hline LII1 - Inferior level of the tank1 & V13 - Cooling of the condenser \\
\hline
\end{tabular}




\begin{tabular}{|l|l|}
\hline $\begin{array}{l}\text { QIS - Electrical conductivity of the } \\
\text { solution in tank1 (concentration) }\end{array}$ & $\begin{array}{l}\text { V15 - Valve of solution passage } \\
\text { of the tank1 for the tank2 }\end{array}$ \\
\hline $\begin{array}{l}\text { TAlarm- Maximum solution } \\
\text { temperature in tank1(sensor T1S1) }\end{array}$ & V16 - Drain of the tank1 \\
\hline LIS2 - Superior level of tank2 & V17 - Heating of the tank2 \\
\hline LII2 - Inferior level of tank2 & V18 - Emptying of the tank2 \\
\hline $\begin{array}{l}\text { TIS2 - Solution temperature in } \\
\text { tank2 }\end{array}$ & $\begin{array}{l}\text { P1 - Emptying pump of the } \\
\text { tank2 }\end{array}$ \\
\hline $\begin{array}{l}\text { FIS - Cooling solution flow of the } \\
\text { condenser C }\end{array}$ & $\begin{array}{l}\text { H1 - Heating Resistance of the } \\
\text { tank1 }\end{array}$ \\
\hline
\end{tabular}

The controller specification was directly translated to Modelica modeling language, more specifically to the library for hierarchical state machines StateGraph [11].

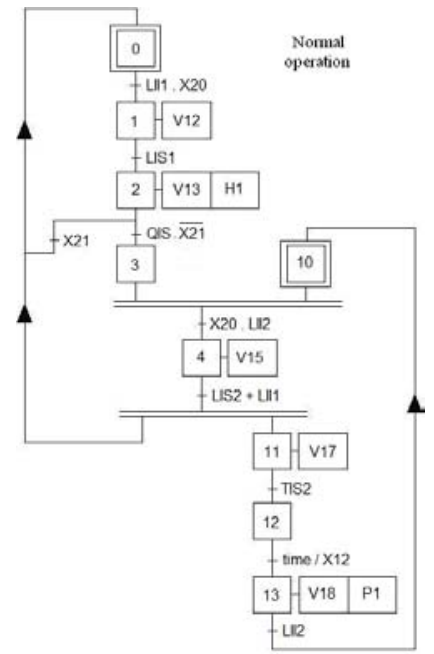

Fig. 3. SFC specification of the Controller - Normal Operation Mode.

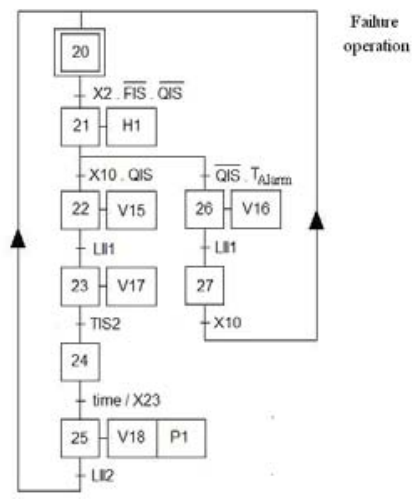

Fig. 4. SFC specification of the Controller - Failure Operation Mode.

\section{Plant Mathematical Modeling}

The plant modelling has two goals: first to assure that the controller specification is adequate for the intended system behaviour and, second, to minimize the cycle time for repetitive automation systems processes. In this paper there are discussed the two of them: to be sure that the system behaves as expected - without leading to dangerous situations - and to maximize the productivity of the process that it implicates the maximization of the number of batches.
The table 2 presents the mathematical equations that model the system.

TABLE II

SYSTEM DESCRIPTION (DIFFERENTIAL AND ALGEBRAIC EQUATIONS)

\begin{tabular}{|c|c|}
\hline $\begin{array}{l}\text { Heating } \\
\text { while } \\
\mathrm{T} 2 \text { is } \\
\text { drained }\end{array}$ & $\begin{array}{l}(d Q / d t)=Q_{\text {Heat }}-Q_{\text {Loss }}-Q_{\text {Evap }} \\
\frac{d H_{1}}{d t}=0 ; \frac{d H_{2}}{d t}=-K_{1} \sqrt{H_{2}} \\
(d Q / d t)=d\left(T \cdot c_{p, L} \cdot\left(m_{L}+m_{V}\right)\right) / d t ; Q_{\text {Loss }}=k A \cdot\left(T-T_{e}\right) \\
Q_{\text {Evap }}=\left(d m_{V} / d t\right) \cdot \Delta h_{e v} ; K_{1}=\left(A_{R} / A_{2}\right) \cdot \sqrt{2 g} \\
p=a_{0}+a_{1} T+a_{2} T^{2} \text { (boiling pressure, dissolve } \\
\text { substance ignored }) \\
p V_{V}=\left(m_{V} / M_{L}\right) R_{m} T ; \Delta h_{e v}=b_{1}+b_{2} T \\
m_{\text {total }}=m_{L}+m_{V}=6 \text { kg (total mass of fluid), } \\
Q H e a t \text { (heat supply rate) } \\
V_{V}=0.02 m^{3} \text { (vapor volume, assumed to be constant), } \\
k A=24 W / K \text { (heat loss flow per Kelvin) }\end{array}$ \\
\hline $\begin{array}{c}\text { Cooling } \\
\text { while } \\
\text { T2 is } \\
\text { drained }\end{array}$ & $\begin{array}{l}(d Q / d t)=-Q_{\text {Loss }}-Q_{\text {Evap }} \\
\frac{d H_{1}}{d t}=0 ; \frac{d H_{2}}{d t}=-K_{1} \sqrt{H_{2}} \\
\mathrm{~T}<373 \mathrm{~K}:(d Q / d t)=d\left(T \cdot c_{p, L .} \cdot m_{L}\right) / d t ; Q_{\text {Evap }} \cong 0 \\
T>373 K, p>1 \text { bar: } \\
(d Q / d t)=d\left(T . c_{p, L .} \cdot\left(m_{L}+m_{V}\right)\right) / d t ; \\
Q_{\text {Evap }}=\left(d m_{V} / d t\right) . \Delta h_{e v} ; Q_{\text {Loss }}=k A .\left(T-T_{e}\right) \\
k A=22.5 W / K \text { (heat loss flow per Kelvin) } \\
\text { Note: In this stage it will be used the same algebraic } \\
\text { equations and parameters as in stage } 1 .\end{array}$ \\
\hline $\begin{array}{l}\text { Stage } 3 \\
\text { Cooling } \\
\text { while } \\
\mathrm{T} 1 \text { is } \\
\text { drained }\end{array}$ & $\begin{array}{l}(d Q / d t)=-Q_{\text {Loss }} \\
\frac{d H_{1}}{d t}=-K_{2} \sqrt{H 1} ; \frac{d H_{2}}{d t}=-K_{1} \sqrt{H_{1}} \\
(d Q / d t)=c_{p, L .} \cdot\left(d T / m_{L}\right) / d t ; Q_{\text {Loss }}=k A \cdot\left(T-T_{e}\right) \\
K_{2}=\left(A_{R} / A_{1}\right) \cdot \sqrt{2 g} ; m_{L}=\rho_{L} H_{1} A_{1} ; A=A_{1}+\pi . D H_{1} \\
k=150 \mathrm{~W} / \mathrm{K} / \mathrm{m}^{2} \text { (heat loss transfer coefficient), } \\
\left.A_{1}=0.03 \mathrm{~m}^{2}, A_{2}=0.06 \mathrm{~m}^{2} \text { (cross-sectional area } \mathrm{T} 1 \text { and } \mathrm{T} 2\right)\end{array}$ \\
\hline Variables & $\begin{array}{l}\text { state: } T \text { (temperature in } \mathrm{T} 1), H_{1}, H_{2} \text { (liquid heights, } \\
\text { tanks considered empty when } H_{1 / 2} \leq 0.0017 m \text { ) } \\
\text { algebraic: } m_{L} \text { (liquid mass), } m_{V} \text { (vapor mass), } \\
\Delta h_{e v} \text { (evaporation enthalpy), } p \text { (pressure), } \\
A \text { (heat loss area) }\end{array}$ \\
\hline $\begin{array}{l}\text { Additional } \\
\text { parameters }\end{array}$ & $\begin{array}{l}A_{1}=0.03 \mathrm{~m} 2, A_{2}=0.06 \mathrm{~m}^{2} \text { (cross-sectional areas of } \\
\mathrm{T} 1 \text { and } \mathrm{T} 2), A_{R}=2.10^{-5} \mathrm{~m}^{2} \text { (pipe cross-sectional area) } \\
a_{0}=9.3 \cdot 10^{6} \mathrm{~N} / \mathrm{m}^{2}, a_{1}=-5.28 \cdot 10^{4} \mathrm{~N} / \mathrm{m}^{2} / \mathrm{K}^{2} \\
a_{2}=75.4 \mathrm{~N} / \mathrm{m}^{2} / \mathrm{K}^{2}\left(a_{0}, a_{1}, a 2 \text { pressure constants), }\right. \\
b_{1}=3.294 \cdot 10^{6} \mathrm{~J} / \mathrm{kg}, b_{2}=-2.78 \cdot 10^{3} \mathrm{~J} / \mathrm{kg} / \mathrm{K} \text { (enthalpy } \\
\text { constant), } c_{p, L}=4220 \mathrm{~J} / \mathrm{kg} / \mathrm{K} \text { (liquid heat capacity), } \\
D=0.2 \mathrm{~m} \text { (diameter of } \mathrm{T} 1), g=9.81 \mathrm{~m} / \mathrm{s}^{2} \text { (gravity } \\
\text { constant), } M_{L}=0.018 \mathrm{~kg} / \mathrm{mol} \text { (molecular weight of } \\
\text { liquid), } \rho_{L}=970 \mathrm{~kg} / \mathrm{m}^{3} \text { (liquid density), } \\
R_{m}=8.314 \mathrm{~J} / \mathrm{kg} / \mathrm{mol} \text { (molecular gas constant), } \\
T_{e}=283 \mathrm{~K} \text { (environment temperature) }\end{array}$ \\
\hline
\end{tabular}


Due to discrete switching between the two different continuous systems (T1 and T2), which happens not only at the stage transitions, by changing the position of the on/off valves (V15 and V18), but also in stage 2 for boiling water point, this developed model is of hybrid nature. The main required parameters and algebraic equations are presented in detail in the table 2 .

The setting of alarm temperature TAlarm is chosen correctly to accomplish the following two opposed very important properties: On the one hand it must be low enough to avoid a dangerous temperature and pressure values, and on the other hand it has to be sufficient high so that temperature $\mathrm{T}$ does not fall below a crystallization temperature before liquid level in tank1 (H1) becomes zero.

\section{MOdelicA MOdEl OF THE SYSTEM}

Due to the described potentialities, it was developed a global model of the evaporator system, already presented in the previous sections. The plant was modeled as the controller using the Dymola software and the object-oriented programming language Modelica [7] [8]. Additionally, to model the controller, it was used the library for hierarchical state machines StateGraph [11], which are included in the Dymola software.

Related with the plant part, it was modeled the filling source, the tank 1 and tank2, the heater (H1), the condenser and the valves. For that, it were used the parameters and algebraic equations presented in the table 2 .

Figure 5 shows the global modelica model of the system, being highlighted the two main parts, the physical part (plant) on the left, and the controller on the right. On the other hand, the controller model was developed according the SFC specifications (see figures 3 and 4).

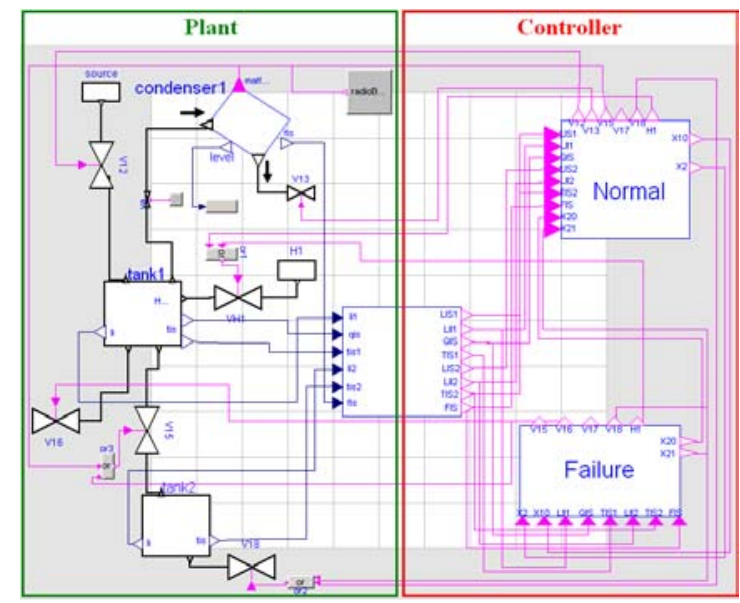

Fig. 5. Global modelica model of the evaporator system.

Also, due to the reason of it being specified a discrete controller to control the hybrid plant, it was necessary to implement an appropriate interface, that translate the analogue outputs signals of the plant (tanks levels, temperatures, concentration,...) digital signals, that can be used as inputs of the discrete controller.

\section{SimULATION RESULTS}

In this section, there are presented results of simulations that were accomplished with the purpose of studying the dynamical behavior of the hybrid models described in the previous sections in order to maximize the productivity of the evaporator process, in terms, of their energy efficiency and batches times.

Moreover, these simulations can be seen as a "system preliminary analysis" to check if the system behaves in agreement to a given specification for a particular case, like as, a given a initial state of the process and a given controller program. However, it must to be enhanced that this is not verification in the strict sense, since it relies on the appropriate selection of the considered cases.

In order to perform the hybrid model simulation with different heating power's it was necessary to define the parameters, start and stop time of the simulation, the interval output length or number of output intervals and the integration algorithm. In the present work, in all simulations performed, the Dassl's integration algorithm [12] with 10000 output intervals was used.

The first simulations performed were devoted to verify if the SFC specification of the controller (see figures 3 and 4) translated to Modelica modeling language, considering the library for hierarchical state machines StateGraph - was able to simulate the evaporator system behavior, according to previously defined, desired and undesired behaviors. It must be highlighted that the simulation involves the modelica model of the controller specification coupled, in a closed-loop configuration, with plant model - modeled also using modelica modeling language - obtained from the equations presented in table II.

Figures 6 and 7 show the results of the first two simulations, respectively, relating to the normal operation and failure operation modes for the level tanks. The failure mode is consequence of the occurrence of the condenser malfunction during the production cycle that it originates that the solution temperature in the tank1 reaches the pre-defined alarm temperature (390K).

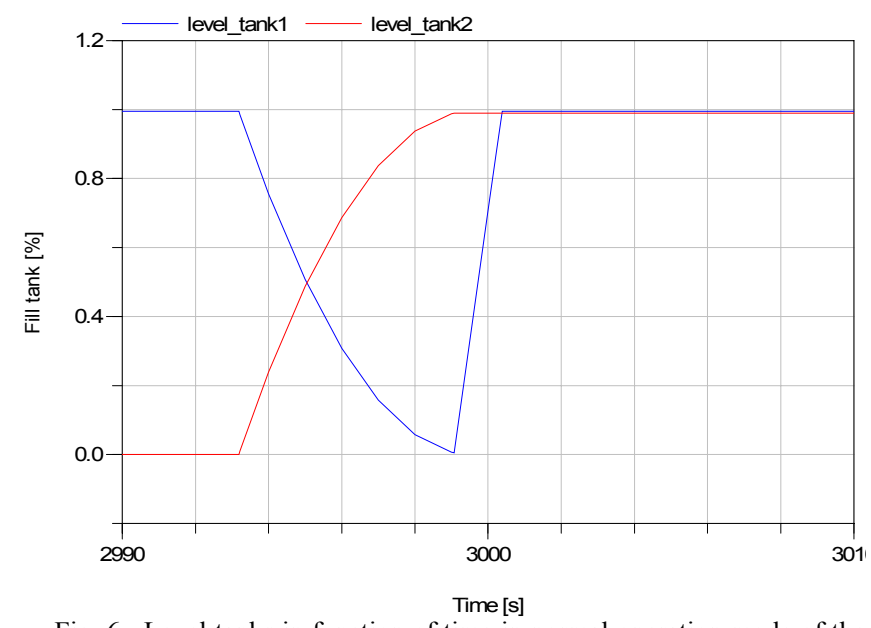

Fig. 6. Level tanks in function of time in normal operation mode of the evaporator system. 


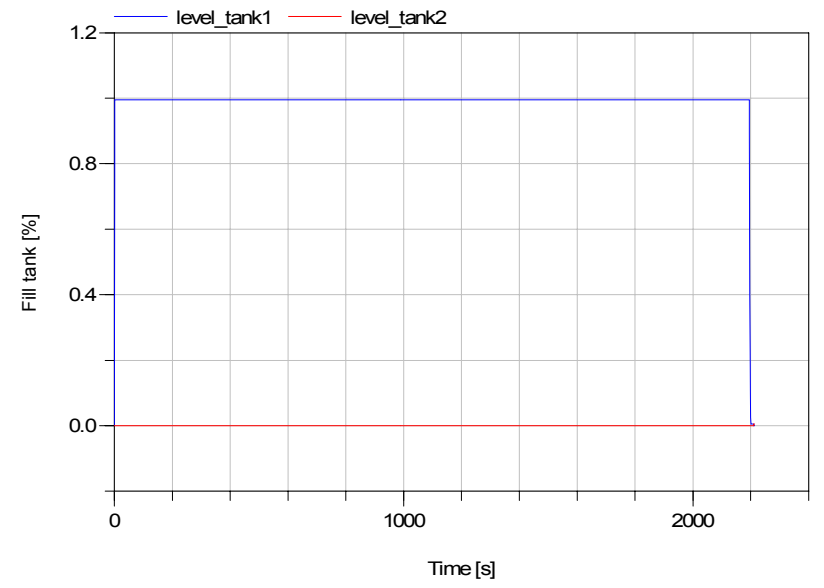

Fig. 7. Level tanks in function of time in failure operation mode of the evaporator system.

Observing Figure 6 it can be concluded that the normal operation mode is properly simulated by the developed program, since the two main properties that are important to prove are confirmed, for instance, the drainage of the solution present in the tank 1 only to happens when the tank 2 is empty and also the filling of the tank1 to happen soon after this to be empty. On the other hand, observing figure 7 , it can be also concluded that the failure operation mode is properly simulated, given that it is proven that the tank1 is drained through the safety valve (V16 - see figure 2) because it is seen that the tank2 remains empty.

After being concluded that the normal and failure operation behavior is properly simulated by the proposed specification they were performed other simulations in order to obtain the relationship between several physical plant parameters that can obtain the best ratio between the number of batches and the supply energy costs.

This manner, among of several physical variables of the process (see table 2) it was chosen the heat supply rate (QHeat) because it is the most relevant variable, that determine the rate of the steam formation (this condenses in the condenser $\mathrm{C}$ ) and correspondingly, the time in that the solution present in the evaporator (tank1) is prepared to be drained (desired concentration reached).

The solution concentration (C) is obtained by the following equation:

$$
C=\left(C_{0} \cdot m_{L}\right) /\left(m_{L}-m_{V}\right)
$$

Where, $\mathrm{C} 0$ is the initial concentration, $\mathrm{mL}$ is the liquid mass and $\mathrm{mV}$ is the vapour mass. In addition, in all of the performed simulations, it was assumed concentration values of 0.01000 and 0.01003 , respectively, initial and final.

Figures 8 and 9 illustrate the behavior of the model given in the table 2 for heat supply rate (QHeat) of $3000 \mathrm{~W}$, respectively for the vapour mass and concentration.

In a general way, the results presented in the figures 8 and 9 allow to conclude that the concentration behavior is properly simulated by the proposed program.

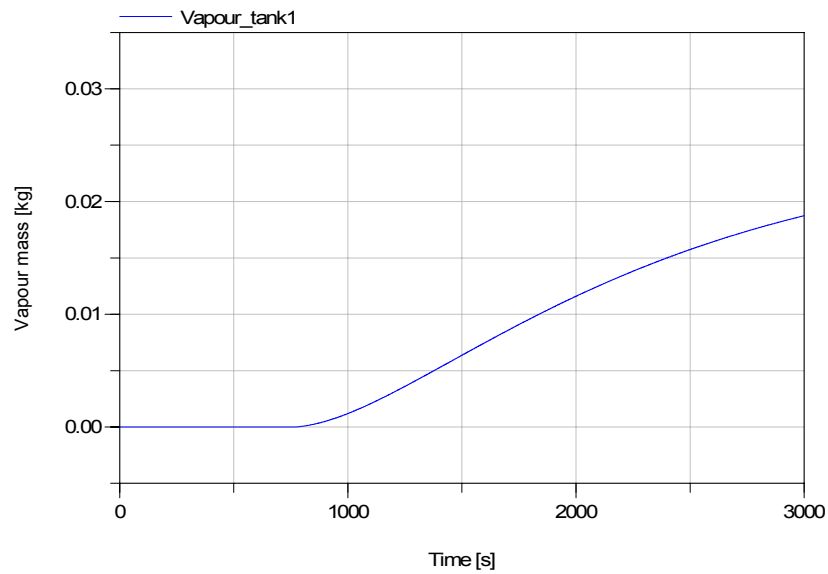

Fig. 8. Vapour mass in function of time with a heat supply rate of $3000 \mathrm{~W}$.

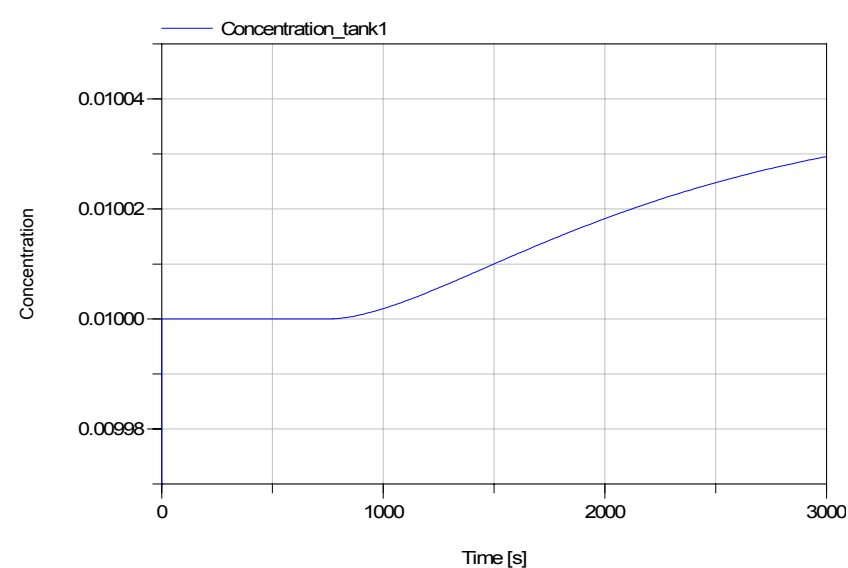

Fig. 9. Concentration in function of time with a heat supply rate of $3000 \mathrm{~W}$.

In particular, analyzing figure 8 it can be stated that the boiling water point $(373 \mathrm{~K})$ it is reached after having elapsed about 800 s and after this time the vapour mass increases continually as it was foreseen with the increase of the temperature.

On other hand, observing figure 9 , it can be verified that the time in that the solution present in the tank1 reaches the final concentration $(0.01003)$, and this way prepared to be drained to tank2, is about $3000 \mathrm{~s}$.

In order to be possible to generalize the batches optimization, that it implicates the productivity maximization of the evaporator system, it is essential to know the optimized relation between the heat supply rate and the time for the solution reaches the desired concentration in the tank1 (evaporator).

Figure 10 illustrates the time for the solution reaches the desired final concentration in function of heat supply rate, as example, from 3000 to $100000 \mathrm{~W}$.

Analyzing figure 10, it can be concluded that the increase of the heat supply rate originates a very significant decrease on the required time for the solution reaches the final concentration. It can be highlighted that the more accentuated time reductions happens in the interval from 3000 to $20000 \mathrm{~W}$. 
This manner, in agreement with the simulations results presented, it can be concluded that the heat supply rate of $20000 \mathrm{~W}$, could be the most appropriate to obtain the best optimization between the number of batches and the supply energy costs, considering the values of the physical variables of the evaporator system presented in table 2 .

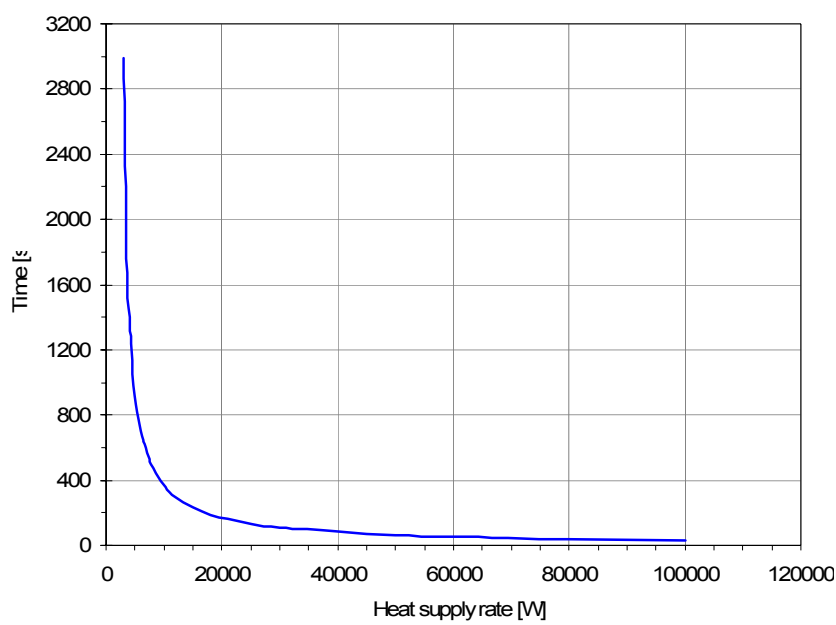

Fig. 10. Time for the solution present in the tank1 reaches the final concentration in function of heat supply rate.

\section{CONCLUSIONS AND FUTURE WORK}

The simulation used to evaluate the controller and plant behavior has been developed and proposed in this paper.

The present research proved to be successful using the Modelica programming Language to obtain a plant model and using it, in a closed-loop behavior, with the controller model.

Some parameters and functional aspects of the system have been simulated in order to define a set of values of different variables that make the system dependable and safe avoiding dangerous situations, and more efficient, when studied some critical plant behavior parameters.

The study of critical plant behavior parameters (like presented in this paper) can be performed using Modelica in order to obtain simulation models of complex systems.

As future work the authors believe that, with auxiliary calculations, it will be possible, using simulation strategies, to define optimal values for the different variables, in order to obtain, by one hand, a safe system behavior and, by other hand, to optimize the time cycle of Automation repetitive systems taking into account the critical steps of their functioning.

\section{ACKNOWLEDGMENTS}

This research project is carried out in the context of the SCAPS Project supported by FCT, the Portuguese Foundation for Science and Technology, and FEDER, the European regional development fund, under contract POCI/EME/61425/2004 that deals with safety control of automated production systems.

\section{REFERENCES}

[1] Karayanakis, N. M.. Advanced System Modelling and Simulation with Block Diagram Languages, CRC Press, Inc, 1995

[2] Matlab, 2010. Matlab Available in http://www.Mathworks.com (consulted in March $05^{\text {th }}, 2010$ ).

[3] MATRIXX, 2010. MATRIXX Available in http://www.ni.com/matrixx (consulted in March $05^{\text {th }}, 2010$ ).

[4] Mosterman P. J,. HYBRSIM - a modeling and simulation environment for hybrid bond graphs. Proceedings of the Institution of Mechanical Engineers, Part I: Journal of Systems and Control Engineering. Vol. 216, $\mathrm{N} 1$, pp 35-46, 2002

[5] MGA Software. ACSL Graphic Modeller - Version 4.1, MGA Software, 1996

[6] Aström, K. J., Elmqvist, H. \& Mattsson, S. E. Evolution of continuous time modeling and simulation, in 'Proceedings of the 12th European Simulation Multiconference', Manchester, UK, pp. 9-18, 1998.

[7] Elmqvist E., Mattson S. An Introduction to the Physical Modelling Language Modelica. Proceedings of the 9th European Simulation Symposium, ESS'97. Passau, Germany, 1997.

[8] Fritzson, Peter, Vadim E.,. Modelica, a general object-oriented language for continuous and discrete event system modeling and simulation, 12th European Conference on Object-Oriented Programming (ECOOP'98). Brussels, Belgium, 1998.

[9] Dymola software. Available in: http://www.3ds.com/products/catia/portfolio/dymola (consulted in Jan $\left.25^{\text {th }}, 2010\right)$.

[10] Kowalewski S., Stursberg O. and Bauer N. An Experimental Batch Plant as a Test Case for the Verication of Hybrid Systems. European Journal of Control. vol. 7, n_4, pp. 400-415, 2001.

[11] Otter M., Årzén K., Dressler I. StateGraph - A Modelica Library for Hierarchical State Machines. Modelica 2005 Proceedings, 2005.

[12] Basu S., Pollack R., Roy M. Algorithms in Real Algebraic Geometry, In Springer (Eds), Algorithms and Computation in Mathematics, (10), 2aedition, 2006. 